\title{
Multi-model Approach for Multicomponent Texture Classification
}

\author{
Ahmed Drissi El Maliani ${ }^{1}$, Mohammed El Hassouni $^{2}$, \\ Yannick Berthoumieu ${ }^{3}$, and Driss Aboutajdine ${ }^{1}$ \\ 1 LRIT, Unité Associée au CNRST (URAC 29), \\ Mohammed V University, Agdal, Morocco \\ 2 DESTEC, FLSHR, Mohammed V University, Agdal, Morocco \\ 3 IMS- Groupe Signal- UMR 5218 CNRS, ENSEIRB, University Bordeaux, France
}

\begin{abstract}
This paper concerns multicomponent texture classification. The aim is to provide a flexible model when wavelet subband coefficients of components do not have the same distributions. Example of such case is when color textures are represented in a perceptual color space. In this kind of representation, the separability between luminance and chrominance components have to be considered in the modeling process. The contribution of this work consists in proposing a multi-model based characterization for this type of multicomponent images. For this, two models $M_{L}$ and $M_{C_{r}}$ are used in order to extract features from luminance and chrominance components, respectively. We discuss in detail and define the multi-model when textures are represented in the HSV color space as a special case of multicomponent analysis. Experimental results show that the proposed approach improves performances of the classification system when compared with existing methods.
\end{abstract}

Keywords: Multicomponent textures, Copula, Rao distance.

\section{Introduction}

Analysis of multicomponent images has become an important and very challenging task with the continuous advance of multimedia tools. Multicomponent image databases are bigger and need to convenient techniques in order to be managed. Many works emphasized that textured images are simple to model in the wavelets domain [8] [9. Distributions of the resulting subbands are characterized using well suited parametric models like generalized Gaussian distribution (GGD) [1] or Weibull distribution [2] This marginal approach is convenient for unicomponent images such as grey level textures. But when retrieval or classification systems deal with multicomponent textures, the dependence across components have to be modeled using multivariate distributions as the multivariate generalized Gaussian distribution (MGGD) [3], or more recently using copula theory [11] 4]. Such multivariate modeling leads to considerable enhancements when compared with the marginal modeling. However, in all aforementioned multivariate studies, the marginal behavior of the different components is not taken 
into account along with modeling dependencies among those components. This fact make the modeling less pertinent, especially when components are separable, i.e do not have the same marginal distributions. An example of such case is when color textures are represented in a luminance-chrominance color space such as HSV (Hue Saturation Value). Luminance-chrominance or more specifically perceptual color spaces represent the most natural and intuitive way to describe color images. Researches on the human visual system revealed that the human eye percepts colors as a luminance and chrominance separated signals, and considers the chrominance as a Hue (pure color information) and saturation (level of intensity of the Hue). Modeling color textures in luminance-chrominance color spaces has been considered by many researchers as in [6] and [7. However, in these works, one given model has been used to describe luminance and chrominance which are considered separable. For this, coming up with a statistical model that takes into account the different natures of luminance and chrominance channels seems to be a welcome advantage. Furthermore, in perceptual color spaces, the existence of a circular component (Hue) must also be taken into account in the feature extraction step.

Based on these assumptions, we propose a multi-model for color texture classification in the HSV color space. Wavelet subbands of the luminance and chrominance are characterized using two independent joint parametric models $M_{L}$ and $M_{C_{r}}$, respectively. These latters repose on copula theory due to its ability to represent different marginals in one joint model which is the case of chrominance components.

\section{Statistical Modeling of Multicomponent Textures}

As said in the introduction, we detail a specific example of multicomponent images which is color textures when represented in HSV color space. In this section, we define the wavelet representation of color textures, we give a review of copulas and define the multi-model (models $M_{L}$ and $M_{C_{r}}$ ).

\subsection{DTCWT Representation of Color Textures in HSV Color Space}

We consider the dual tree complex wavelet transfrom (DTCWT) in order to overcome disadvantages of the classic discrete wavelet transform (DWT) decomposition. DWT suffers from the lack of shift-invariance and directional selectivity, since it provides only three orientations at each decomposition level. DTCWT is based on two real wavelets to resort with complex wavelet coefficients, which will be shift-invariant. DTCWT provides six detail subbands per scale instead of three subbands in the case of DWT, which presents a rich directional selectivity.

Let us suppose a color texture $I_{M}$ from the database. $I_{M}$ is represented in the HSV perceptual color space. Let $l, h$ and st, be the luminance (value or brightness), hue and saturation components of $I_{M}$ respectively. We decompose each of those components via a DTCWT, and we call $l_{k}=l_{s ; o}, h_{k}=h_{s ; o}$, $s t_{k}=s t_{s ; o}$ the wavelet subbands in scale $\mathrm{s}$ and orientation o respectively for the three components. 


\subsection{Copulas Theory}

A copula is a multivariate cumulative distribution function (cdf), defined on the d-dimensional unite cube $[0 ; 1]^{d}$. Given a d-dimensional vector $X=\left[X_{1}, \ldots, X_{d}\right]$ on the unit cube $[0 ; 1]$, with a cumulative distribution function $\mathrm{F}$ and marginal distributions $F_{1}, \ldots, F_{d}$ Sklar theorem [10] shows that there exist a d-dimensional copula $\mathrm{C}$ such that:

$$
F\left(x_{1}, \ldots, x_{d}\right)=C\left(F_{1}\left(x_{1}\right), \ldots, F_{d}\left(x_{d}\right)\right)
$$

The joint PDF is then deduced uniquely from the margins and the copula density (dependence structure) as follows:

$$
f\left(x_{1}, \ldots, x_{d}\right)=c\left(F_{1}\left(x_{1}\right), \ldots, F_{d}\left(x_{d}\right)\right) \prod_{i=1}^{d} f_{i}\left(x_{i}\right)
$$

where $f_{i}, i=1, \ldots, d$, represent the marginal densities.

We use the Gaussian copula for its advantages in term of computation and naturality of the dependence structure. Copula density $c_{\phi}$ is then defined by:

$$
c_{\phi}(u, \Sigma)=\frac{1}{|\Sigma|^{1 / 2}} \exp \left[-\frac{1}{2} \vartheta^{T}\left(\Sigma^{-1}-I\right) \vartheta\right]
$$

with $\vartheta_{i}=\phi^{-1}\left(F_{i}\left(u_{i}\right)\right)$, and $\phi$ represents the standard normal cumulative distribution function. $\Sigma$ denotes the correlation matrix, and $I$ denotes the ddimensional matrix identity.

To estimate parameters of copula based models, we use the Inference From Margins (IFM) method [12].

\subsection{Multivariate Model for Luminance}

We study the spatial structure information for luminance via the model $M_{L}$. The dataset representing the neighborhood to be modeled by $M_{L}$ is constructed from each subband $l_{k}$ by moving a window of size $d=(2 p+1) \times(2 q+1)$ in an overlapping manner. Assuming the spatial homogeneity of subbands, we start from a reference coefficient $l_{k}(i, j)$ from the $k_{t h}$ subband $l_{k}$, and then we concatenate neighbors to have:

$$
l_{k}=\left[l_{k}(i-p, i-q), \ldots, l_{k}(i+p, i+q)\right]^{T}
$$

Based on the Gaussian copula, the model $M_{L}$ is defined by choosing appropriate pdf as a marginal for luminance:

$$
f_{M_{L}}(l)=c_{\phi}\left(F_{1}\left(l_{1}\right), F_{2}\left(l_{2}\right), \ldots, F_{2}\left(l_{N}\right)\right) \prod_{i=1}^{N} f_{i}\left(l_{i}\right)
$$

that is:

$$
f_{M_{l}}(l ; w, \Sigma)=\frac{1}{|\Sigma|^{1 / 2}} \exp \left[-\frac{1}{2} \vartheta^{T}\left(\Sigma^{-1}-I\right) \vartheta\right] \prod_{i=1}^{N} f_{i}\left(l_{i}, w_{i}\right)
$$


where $w=\left(w_{1}, w_{2}, \ldots, w_{N}\right)$ denotes the parameters of luminance marginals, and $N$ represents the number of detail subbands.

\subsection{Bivariate Model for Chrominance}

We model the chrominance by a bivariate model representing the correlation between chrominance subbands. Given the $k_{t h}$ subband, this dependency is represented by 2 -dimensional vector $c_{r}{ }^{(k)}$ as $c_{r}{ }^{(k)}=\left[h_{k}, s t_{k}\right]$. The model $M_{C_{r}}$ is also defined reposing on the Gaussian copula:

$$
f_{M_{C_{r}}}\left(c_{r}\right)=c_{\phi}\left(F_{1}(h), F_{2}(s t)\right) f_{1}(h) f_{2}(s t)
$$

that is:

$$
f_{M_{c_{r}}}\left(c_{r} ; w, \Sigma\right)=\frac{1}{|\Sigma|^{1 / 2}} \exp \left[-\frac{1}{2} \vartheta^{T}\left(\Sigma^{-1}-I\right) \vartheta\right] \prod_{i=1}^{2} f_{i}\left(c_{i}, w_{i}\right)
$$

where $w=\left(w_{1}, w_{2}\right)$ the parameters of chrominance components margins, and $\Sigma$ the correlation matrix.

\section{Classification Results}

\subsection{Experimental Setup}

Texture classification was chosen as an application in order to validate the proposed multi-model. Experiment was carried out on 24 textures from the Vistex database [14] as shown in [7]. Each image of size $512 \times 512$ was divided into subimages of size $32 \times 32$ pixels. Then for each image, we consider 96 from the resulting 256 subimages as the training set, while the remaining 160 subimages are considered as the test set. For all textures of our database, every component of each subimage was normalized by subtracting its mean and dividing by its standard deviation, and then decomposed using a 2 scales DTCWT with a Q-shift $(14,14)$ tap filter. Here, we use the K-nearest neighbor classifier which is the most simple and straightforward classification method. KNN is a kind of instance based classifiers, where the main idea is that an instance is classified reposing on a similarity measure, and is accorded the label of the majority of its K-nearest neighbor. Thus we need to a pertinent similarity measure that accounts of the multi-model approach.

\subsection{Similarity Measurement Based on Rao Distance}

Measuring similarity between two color textures in the database is done by measuring closeness of there luminance and chrominance components individually according to a luminance and a chrominance Rao distances, and then by calculating the overall similarity measure between color textures using a combination method as :

$$
L=\lambda L_{L}+(1-\lambda) L_{C_{r}}
$$


where $L_{L}$ represents the Rao distance between luminance models, and $L_{C_{r}}$ the Rao distance between chrominance models. The coefficient $\lambda$ is calculated empirically by considering the learning set as the test set and then returning value of $\lambda$ that leads to the best classification rates. In [5], we determined a closed form expression of Rao distance between two copula based pdfs as the sum of the Rao distances between the two copulas and the Rao distances between the marginal distributions. Thus, the Rao distance between two copulas based probability density functions $f\left(x ; \theta_{1}\right)$ and $f\left(x ; \theta_{2}\right)$ is defined as follows:

$$
\begin{gathered}
L\left(f\left(x ; \theta_{1}\right) \| f\left(x ; \theta_{2}\right)\right)=L_{\text {Gauss }}\left(f\left(x ; \Sigma_{1}\right) \| f\left(x ; \Sigma_{2}\right)\right)+ \\
\sum_{i=1}^{d} L_{\text {Margins }}\left(f\left(x ; w^{(1)}\right) \| f\left(x ; w^{(2)}\right)\right)
\end{gathered}
$$

that is:

$L\left(f\left(x ; \theta_{1}\right) \| f\left(x ; \theta_{2}\right)\right)=\left[\frac{1}{2} \sum_{i=1}^{d}\left(\ln r^{i}\right)^{2}\right]^{1 / 2}+\sum_{i=1}^{d} L_{\text {Margins }}\left(f\left(x ; w^{(1)}\right) \| f\left(x ; w^{(2)}\right)\right)$

where $r^{i}, i=1, \ldots, d$ represents the eigenvalues of $\Sigma_{1}^{-1} \Sigma_{2}$.

Expressions of $L_{\text {Margins }}$ for Weibull and Gamma marginals were provided in [5] [16], for the vonMises marginal expression of the Rao distance can be found in $[15]$.

\subsection{The Multi-model}

To come up with most pertinent multi-model for the multicomponent textures, we test different combinations of luminance and chrominance models. We use copula based multivariate Gamma and copula based multivariate Weibull models for luminance, beside the copula based bivariate models \{vonMises, Weibull and $\{$ vonMises, Gamma $\}$ for chrominance.

\section{-Model $M_{L}$}

From equation (9), if we consider Gamma marginals for luminance, the pdf of $M_{L}$ is presented as follows:

$f_{M_{L}}(x, \theta)=\frac{1}{|\Sigma|^{1 / 2}} \exp \left[-\frac{1}{2} \vartheta^{T}\left(\Sigma^{-1}-I\right) \vartheta\right] \times\left(\frac{\beta^{-\alpha}}{\Gamma(\alpha)}\right)^{d} \prod_{i=1}^{d} x_{i}^{\alpha-1} \exp -\sum_{i=1}^{d}\left(\frac{x_{i}}{\beta}\right)$

with $\theta=(\alpha, \beta, \Sigma)$, $\alpha$ represents the shape parameter, $\beta$ represents the scale parameter, and $\Sigma$ denotes the covariance matrix. We call this model the copula based multivariate Gamma (CopMGam).

When, we consider Weibull marginals, the pdf of $M_{L}$ is presented by:

$$
f_{M_{L}}(x, \theta)=\frac{1}{|\Sigma|^{1 / 2}} \exp \left[-\frac{1}{2} \vartheta^{T}\left(\Sigma^{-1}-I\right) \vartheta\right] \times\left(\frac{\tau}{\lambda}\right)^{d} \prod_{i=1}^{d} x_{i}^{\tau-1} \exp -\sum_{i=1}^{d}\left(\frac{x_{i}}{\lambda}\right)^{\tau}
$$




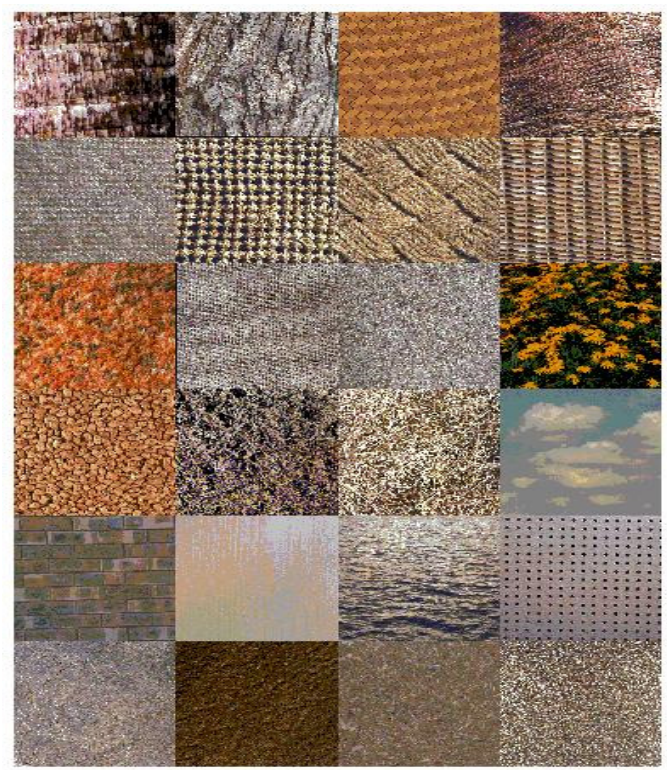

Fig. 1. 24 texture classes from Vistex database

with $\theta=(\tau, \lambda, \Sigma), \tau$ represents the shape parameter, $\lambda$ represents the scale parameter, and $\Sigma$ denotes the covariance matrix. In this case, we call the model $M_{L}$ as copula based multivariate Weibull ( $\left.C o p M W b l\right)$.

-Model $M_{C_{r}}$

As already said, the chrominance model $M_{C_{r}}$ accounts for the different natures of chrominance components. For this we use the property of merging different marginals when the model is based on copulas. We use two different marginals for the Hue and the Saturation components respectively. Then we use the Gaussian copula for the dependence structure. We test two couples of chrominance marginals.

Couple $\{$ vonMises, Weibull\}, means that we use vonMises distribution for the Hue and Weibull for the Saturation. Thus, the probability density of the joint linear-circular model $M_{C_{r}}$ is as follows:

$$
\begin{gathered}
f_{M_{C_{r}}}(x, \theta)=\frac{1}{|\Sigma|^{1 / 2}} \exp \left[-\frac{1}{2} \vartheta^{T}\left(\Sigma^{-1}-I\right) \vartheta\right] \times \\
\frac{\tau}{2 \pi \lambda I_{0}(\nu)}\left(\frac{x_{2}}{\lambda}\right)^{\tau-1} \exp \left[\nu \cos \left(x_{1}-\mu\right)-\left(\frac{x_{2}}{\lambda}\right)^{\tau}\right]
\end{gathered}
$$


where $\theta=(\mu, \nu, \tau, \lambda, \Sigma)$ the hyperparameters of the chrominance model. $\mu$ and $\nu$, are respectively the mean direction and the concentration parameters for the vonMises marginal, while $\tau$ and $\lambda$ are respectively the shape and scale parameters of the Weibull marginal, $\Sigma$ represents the covariance matrix.

If we consider $\{$ vonMises, Gamma\}, the probability density of the joint linearcircular model $M_{C_{r}}$ is defined as:

$$
\begin{aligned}
& f_{M_{C_{r}}}(x, \theta)=\frac{1}{|\Sigma|^{1 / 2}} \exp \left[-\frac{1}{2} \vartheta^{T}\left(\Sigma^{-1}-I\right) \vartheta\right] \times \\
& \frac{\beta^{-\alpha}}{2 \pi \Gamma(\alpha) I_{0}(\nu)}\left(\frac{x_{2}}{\beta}\right)^{\alpha-1} \exp \left[\nu \cos \left(x_{1}-\mu\right)-\left(\frac{x_{2}}{\beta}\right)^{\alpha}\right]
\end{aligned}
$$

where $\theta=(\mu, \nu, \alpha, \beta, \Sigma)$ the hyperparameters of the chrominance model. $\mu$ and $\nu$, are respectively the mean direction and the concentration parameters for the vonMises marginal, while $\alpha$ and $\beta$ are respectively the shape and scale parameters of the Gamma marginal, $\Sigma$ represents the covariance matrix.

\subsection{Results}

We present results for different combinations of the multi-model:

- multi-model 1: CopMGam for luminance and the $\{$ vonMises, Weibull $\}$ for chrominance.

- multi-model 2: CopMWbl for luminance and the $\{$ vonMises,Weibull $\}$ for chrominance.

- multi-model 3: CopMGam for luminance and the \{vonMises,Gamma\} for chrominance.

- multi-model 4: CopMWbl for luminance and the \{vonMises,Gamma\} for chrominance.

Table 1, presents percentage classification of color textures using the four combinations of the multi-model in comparison with the MGGD based approach [3] and the copula based joint Weibull approach [4] in the RGB color space. It is to note that we consider the same aforementioned experience conditions for all methods. We can clearly observe from these results that considering multi-model for both luminance and chrominance information, beside accounting for the circular Hue component improves the classification rates, when compared with the uni modeling approach wether when components are characterized using MGGD or copula based joint Weibull models. A percentage classification of $94.37 \%$ is achieved using multi-model 1 , while when luminance and chrominance components are characterized by the same model the rates are just $89.74 \%$ and $91.87 \%$ for models proposed in [3] and [4] respectively. We also deduce from Table 1 that the multi-model 1 leads to better rates, this is due to the ability of CopMGam in modeling spatial structure as stressed in [13] and the suitability of Weibul marginal for characterizing the Saturation component. 
Table 1. Average classification rate using the multi-model method in comparison with existing approaches

\begin{tabular}{|c|c|}
\hline Approach & Percentage classification (\%) \\
\hline multi-model 1 & 94.37 \\
\hline multi-model 2 & 93.48 \\
\hline multi-model 3 & 93.95 \\
\hline multi-model 4 & 93.15 \\
\hline MGGD/RGB [3] & 89.74 \\
\hline CopWbl/RGB [4] & 91.87 \\
\hline
\end{tabular}

\section{Conclusion}

We proposed a multi-model characterization for multicomponent images and more specifically for color textures in perceptual color spaces. A model for luminance and another model for chrominance were used to consider the separability of these latters in such color spaces. We have also taken into account the angular nature of the Hue component in the modeling process. Results on the Vistex database show the superiority of the proposed approach in comparison with the existing statistical modeling approaches.

\section{References}

1. Do, M., Vetterli, M.: Wavelet-based texture retrieval using generalized Gaussian density and Kullback-Leibler distance. IEEE Transactions on Image Processing 11, 146-158 (2002)

2. Kwitt, R., Uhl, A.: Image similarity measurement by Kullback-Leibler divergences between complex wavelet subband statistics for texture retrieval. In: 15th IEEE International Conference on Image Processing, ICIP 2008, pp. 933-936 (2008)

3. Verdoolaege, G., Scheunders, P.: Geodesics on the manifold of multivariate generalized gaussian distributions with an application to multicomponent texture discrimination. Int. J. Comput. Vision 95, 265-286 (2011)

4. Kwitt, R., Meerwald, P., Uhl, A.: Efficient texture Image Retrieval Using Copulas in a Bayesian Framework. IEEE Transactions on Image Processing 20, 2063-2077 (2010)

5. El Maliani, A.D., El Hassouni, M., Lasmar, N.-E., Berthoumieu, Y., Aboutajdine, D.: Color Texture Classification Using Rao Distance between Multivariate Copula Based Models. In: Real, P., Diaz-Pernil, D., Molina-Abril, H., Berciano, A., Kropatsch, W. (eds.) CAIP 2011, Part II. LNCS, vol. 6855, pp. 498-505. Springer, Heidelberg (2011)

6. Kato, Z., Pong, T.C.: A Markov random field image segmentation model for color textured images. Image and Vision Computing 24(10), 1103-1114 (2006)

7. Qazi, I.U.H., Alata, O., Burie, J.C., Fernandez- Maloigne, C.: Color spectral analysis for spatial structure characterization of textures in IHLS color space. Pattern Recognition 43(3), 663-675 (2010)

8. Mallat, S.: A Wavelet Tour of Signal Processing, 3rd edn. The Sparse Way. Academic Press (2008) 
9. Manjunath, B.S., Ma, W.Y.: Texture features for browsing and retrieval of image data. IEEE Trans. Pattern Anal. Mach. Intell. 18, 837-842 (1996)

10. Sklar, M.: Fonctions de répartition à $n$ dimensions et leurs marges. Publications de l'institut de Statistique de l'Université de Paris 8, 229-231 (1959)

11. Sakji-Nsibi, S., Benazza-Benyahia, A.: Fast scalable retrieval of multispectral images with kullback-leibler divergence. In: Proceedings of the 17th IEEE Interational Conference on Image Processing (ICIP 2010), Hong Kong, pp. 2333-2336 (2010)

12. Joe, H.: Multivariate Models and Dependence Concepts. Monographs on Statistics and Applied Probability. Chapman \& Hall (1997)

13. Stitou, Y., Berthoumieu, Y., Lasmar, N.: Copulas based multivariate gamma modeling for texture classification. In: IEEE International Conference on Acoustics, Speech and Signal Processing, ICASSP 2009, Taipei, Taiwan, April 19-24, pp. 1045-1048 (2009)

14. MIT vision and modeling group, http://vismod.media.mit.edu

15. Ceolin, S., Hancock, E.R.: Characterising facial gender difference using fisherrao metric. In: Proceedings of the 2010 20th International Conference on Pattern Recognition, ICPR 2010, pp. 4308-4311 (2010)

16. Reverter, F., Oller, J.M.: Computing the Rao distance for Gamma distributions. Journal of Computational and Applied Mathematics 157, 155-167 (2003) 